\title{
PRICE COMPETITION STRATEGY OF INTERNET PLATFORMS
}

\author{
L. ZHUANG \\ School of Economics and Management, Southeast University, China.
}

\begin{abstract}
This paper generalizes the economic features of internet platforms and analyses their competitive strategies with the method of insulating pricing. The analysis focuses on the quantity expectations game of platforms and their price expectations game. The function of network externalities on the internet platforms has an obvious feature of two stages, so their competitive strategies have to be divided into two distinct phases. Before the critical mass stage, companies need to pursue a rapid increase of subscribers in order to survive. After the critical capacity, differentiation strategy can be implemented by platform companies which seek maximum profit by the action of network externalities.

Keywords: competitive strategy, insulating pricing, internet platform, non-cooperative game.
\end{abstract}

\section{INTRODUCTION}

In the internet era of big data, various internet platforms stake to fierce competition, which leads to a problem about how the internet platforms compete. Compared with the traditional competitive strategies, competitive strategy of internet platforms hides the key factor to success [1,2]. This article attempts to explain competitive strategy of internet platforms from a perspective of the network economic, which provides theoretical support and practical guidance for competition on the internet platforms [3].

Internet platforms are the product of network economic development, so they have a lot of different characteristics compared with traditional companies [4]. First, platforms have significant network externalities [5], which are generally divided into membership and usage externalities [6, 7]. In addition to the analysis of network effects of platforms [8], more literature discusses pricing strategy of platforms from membership externalities and usage externalities [9]. Weyl [10] analyses optimal pricing of platforms under different targets with the method of insulating tariffs [11]. A more realistic problem is how platforms participate in the competition [12]. Hence, the paper mainly analyses the competitive strategies of internet platforms through two-sided markets and game theory.

This article is structured as follows: Section 1 introduces the basic features and model assumptions of the internet platforms. Section 2 constructs theoretical models of platforms competition, includes the dynamic model about users' scale expectations and price expectations. Section 3 summarizes the full paper and makes some relevant suggestions.

\section{THEORETICAL MODEL}

To analyze competitive strategies of platforms more clearly, this article gives some assumptions:

(1) This analysis focuses on two platforms $(i, j)$. When an internet platform makes decisions, the other platforms are considered as a whole by it, namely two platforms in the market. Platforms have two sides, $d$ side and $s$ side.

(2) Only considering cross externalities of platforms, without considering externalities in the same side of platforms. Because the role of network makes marginal cost providing services to users is close to zero. 
2.1 Dynamic game model of quantity expectations

First, we analyze how bilateral platforms take price competition with the methods of insulating tariffs and Game of expectation about users' quantity or scale (as amended Cournot model). Internet platforms have cross network externalities. The price of one side by platform charged is based on the (expected) quantity of users on the other size [13].

Therefore, the pricing function of competitive platforms is as follows:

$$
\left\{\begin{array} { l } 
{ p _ { i } ^ { d } ( n _ { i } ^ { s } ) = \alpha _ { i } ^ { d } n _ { i } ^ { s } + h _ { i } n _ { j } ^ { d e } } \\
{ p _ { i } ^ { s } ( n _ { i } ^ { d } ) = \alpha _ { i } ^ { a } n _ { i } ^ { d } + h _ { i } n _ { j } ^ { s e } ; }
\end{array} \left\{\begin{array}{l}
p_{j}^{d}\left(n_{i}^{s}\right)=a_{j}^{d} n_{j}^{s}+h_{j} n_{i}^{d e} \\
p_{j}^{s}\left(n_{j}^{d}\right)=a_{j}^{s} n_{j}^{d}+h_{j} n_{i}^{s e}
\end{array}\right.\right.
$$

where, $h_{i} n_{n}{ }^{d e}$ is the competitors' market share of a platform and $h_{i}$ is the adjustment factor which can be either positive or negative, but it is usually negative in the model about expected quantity. It means that the price of the platform charged and user scale of the other side are negatively correlated.

Profit function of platforms can be transformed into:

$$
\left\{\begin{array}{l}
\pi_{i}=\left(\alpha_{i}^{d} n_{i}^{s}+h_{i} n_{j}^{d e}-c_{i}^{d}\right) n_{i}^{d}+\left(\alpha_{i}^{s} n_{i}^{d}+h_{i} n_{j}^{s e}-c_{i}^{s}\right) n_{i}^{s}-C_{i} \\
\pi_{j}=\left(\alpha_{j}^{d} n_{j}^{s}+h_{j} n_{i}^{d e}-c_{j}^{d}\right) n_{j}^{d}+\left(\alpha_{j}^{s} n_{j}^{d}+h_{j} n_{i}^{s e}-c_{j}^{s}\right) n_{i}^{s}-C_{j}
\end{array}\right.
$$

Proposition 1: In the game about expected quantity, the price of one side set by platforms is determined by cross network externalities and expected adjustment of competitors' market scale. Final equilibrium results are:

$$
\left\{\begin{array} { l } 
{ n _ { i } ^ { d } = \frac { c _ { i } ^ { s } - h _ { i } n _ { j } ^ { s e } } { a _ { i } ^ { d } + a _ { i } ^ { s } } } \\
{ n _ { i } ^ { s } = \frac { c _ { i } ^ { d } - h _ { i } n _ { j } ^ { d e } } { a _ { i } ^ { d } + a _ { i } ^ { s } } } \\
{ n _ { j } ^ { d } = \frac { c _ { j } ^ { s } - h _ { j } n _ { i } ^ { s e } } { a _ { j } ^ { d } + a _ { j } ^ { s } } } \\
{ n _ { j } ^ { s } = \frac { c _ { j } ^ { d } - h _ { j } n _ { i } ^ { d e } } { a _ { j } ^ { d } + a _ { j } ^ { s } } ; }
\end{array} \left\{\begin{array}{l}
p_{i}^{d}=\frac{a_{i}^{d} c_{i}^{d}+a_{i}^{s} h_{i} n_{j}^{d e}}{a_{i}^{d}+a_{i}^{s}} \\
p_{j}^{d}=\frac{a_{i}^{s} c_{i}^{s}+a_{i}^{d} h_{i} n_{j}^{s e}}{a_{i}^{d}+a_{i}^{s}} \\
p_{j}^{s} c_{j}^{d}+a_{j}^{s} h_{j} n_{i}^{d e} \\
a_{j}^{d}+a_{j}^{s}
\end{array}\right.\right.
$$

From the result, the users' scale of one side on the platform is decided by marginal cost of providing theses service, target users' scale of competitors and cross network externalities.

The price of one side on the platform is depended on cross network externalities, the expected share of competitors and marginal cost.

Then, we analyze the impact of each factor on the platform pricing using the method of control variables: 
(1) Marginal cost. Assuming that the cross network externalities coefficient and the expected scale of competitors are constant, the price function is:

$$
p_{i}^{d}=\frac{\overline{a_{i}^{d}} c_{i}^{d}+\overline{a_{i}^{s}} \overline{h_{i} n_{j}^{d e}}}{\overline{a_{i}^{d}}+\overline{a_{i}^{s}}} .
$$

It means that the price set by platforms is a positive correlation with the marginal cost of platforms' services. This is consistent with the law of economics that the higher the cost of these services provided for each customer interaction, the higher the price charged by platforms.

(2) Expected scale of competitors'other users. Assuming that cross network externalities coefficient and marginal cost are constant, the price function is:

$$
p_{i}^{d}=\frac{\overline{a_{i}^{d}} c_{i}^{d}+\overline{a_{i}^{s}} h_{i} n_{j}^{d e}}{\overline{a_{i}^{d}}+\overline{a_{i}^{s}}}\left(\text { where } h_{i}<0\right) .
$$

It means that the price set by platforms is a negative correlation with the expected scale of users on the other side of other competing platforms. This is consistent with the law of economics that the more the expected scale of users on the other side of other competing platforms, the lower the price charged by platforms.

(3) Cross network externalities coefficient. Assuming that the expected scale of competitors and marginal cost are constant, the price function is:

$$
p_{i}^{d}=\frac{a_{i}^{d} \overline{c_{i}^{d}}+a_{i}^{s} \overline{h_{i} n_{j}^{d e}}}{a_{i}^{d}+a_{i}^{s}}\left(\text { where } h_{i}<0\right) .
$$

When $c_{i}^{d}>h_{i} n_{j}^{d e}$ cross network externalities coefficient $\alpha_{i}^{d}$ is a positive correlation with the price charged by platforms, and cross network externalities coefficient $a_{i}^{s}$ is a negative correlation with the price charged by platforms. When $c_{i}^{d}<h_{i} n_{j}^{d e}$ cross network externalities coefficient $\alpha_{i}^{d}$ is a negative correlation with the price charged by platforms, and cross network externalities coefficient $\alpha_{i}^{s}$ is a positive correlation with the price charged by platforms.

When the scale of platforms exceeds critical capacity, marginal cost of the service provided by platforms is close to zero, $c_{i}^{d}=0$. Then,

$$
p_{i}^{d}=\frac{a_{i}^{s} \overline{h_{i} n_{j}^{d e}}}{a_{i}^{d}+\alpha_{i}^{s}},
$$

which means that the price is mainly influenced by cross network externalities coefficient. When the scale of one side on the platform $i$ increases gradually and makes $c_{i}^{d}>h_{i} n_{j}^{d e}$ the competition of platforms can reach an equilibrium finally.

Thus, internet platforms has an obvious feature of two stages. After the scale of users exceeds critical capacity, platforms can increase the pricing for service based on the size of externalities, and then enter the competitive differentiation stage. 
Corollary 1: In repeated games, the equilibrium prices for internet platforms are:

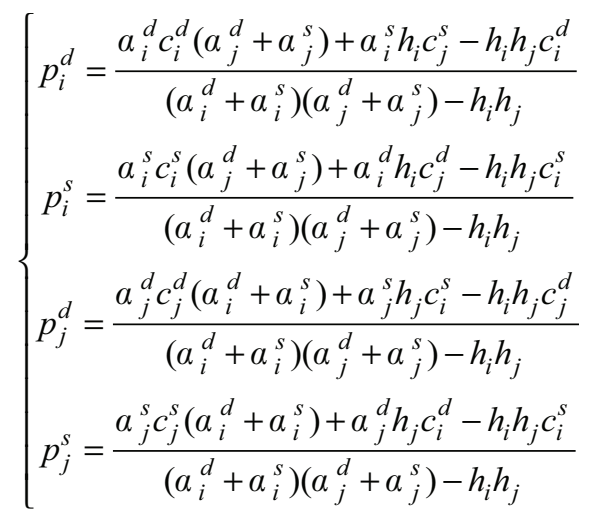

So, these competing platforms can reach an equilibrium state under the condition that platforms set a price based on the coefficient of interactive value in the game of quantity expectations.

To simplify the analysis, the interaction coefficient and costs of the same platform do not distinguish, namely $a_{i}^{d}=a_{i}^{s}=a_{i}, c_{i}^{d}=c_{i}^{s}=c_{i}, c_{j}^{d}=c_{j}^{s}=c_{j}$. Thus, the price function can be simplified as

$$
p_{i}=\frac{2 a_{i} c_{i} \overline{a_{j}}+a_{i} h_{i} \overline{c_{j}}-h_{i} \overline{h_{j}} c_{i}}{4 a_{i} \overline{a_{j}}-h_{i} \overline{h_{j}}}
$$

The price on the side set by platforms depends on the interaction coefficient, marginal cost factor and the expected users' share of opponents.

Partial derivative on the price factor is as follows:

$$
\left\{\begin{array}{l}
\frac{\partial p_{i}}{\partial \alpha_{i}}=\frac{\left(2 \overline{a_{j}} c_{i}-h_{i} \overline{c_{j}}\right) h_{i} \overline{h_{j}}}{\left(4 a_{i} \overline{a_{j}}-h_{i} \overline{h_{j}}\right)^{2}} \\
\frac{\partial p_{i}}{\partial c_{i}}=\frac{2 a_{i} \overline{a_{j}}-h_{i} \overline{h_{j}}}{\left(4 a_{i} \overline{a_{j}}-h_{i} \overline{h_{j}}\right)} \\
\frac{\partial p_{i}}{\partial h_{i}}=\frac{2 a_{i} \overline{a_{j}}\left(2 a_{i} \overline{c_{j}}-c_{i} \overline{h_{j}}\right)}{\left(4 a_{i} \overline{a_{j}}-h_{i} \overline{h_{j}}\right)^{2}}
\end{array}\right.
$$

(1) Expected scale of competitors. When

$$
a_{i}>\frac{c_{i}}{2}, \frac{\partial p_{i}}{\partial h_{i}}>0
$$

the price and expected share are a positive correlation. When

$$
a_{i}<\frac{c_{i}}{2}, \frac{\partial p_{i}}{\partial h_{i}}<0,
$$


the price and expected share are a negative correlation.

(2) Cross network externalities coefficient. When

$$
c_{i}^{d}>h_{i}, \frac{\partial p_{i}}{\partial \alpha_{i}}>0,
$$

the price and cross network externalities coefficient are a positive correlation. When

$$
c_{i}^{d}<h_{i}, \frac{\partial p_{i}}{\partial \alpha_{i}}<0,
$$

the price and cross network externalities coefficient are a negative correlation.

These results further prove the existence and impact of critical mass in the platforms network. When

$$
a_{i}=\frac{c_{i}}{2},
$$

the platforms network reaches a critical mass.

\subsection{Dynamic game model of price expectations}

This article analyzes the competitive behavior of internet platforms from the angle of expected quantity games strategy previously. However, it is difficult to observe user scale of competitors in reality when platforms make specific decisions. Therefore, people concern more about competitors' recent price strategy. Then, the paper further analyzes competitive behavior of internet platforms from the angle of expected price games strategy. According to adaptive expectations in economics (time nearly effects of business or personal decisions), we amend competition model of platforms with joining the recent price adjustment factor.

Assuming price adjustment factor of platforms is influenced by the price of competitors in the previous period. The pricing function of platforms is

$$
\left\{\begin{array}{l}
p_{i t}^{d}\left(n_{i t}^{s}, p_{j(t-)}^{d}\right)=a_{i}^{d} n_{i t}^{s}+h_{i}^{d} p_{j(t-1)}^{d} \\
p_{i t}^{s}\left(n_{i t}^{d}, p_{j(t-)}^{s}\right)=a_{i}^{s} n_{i t}^{d}+h_{i}^{s} p_{j(t-1)}^{s}
\end{array},\left\{\begin{array}{l}
p_{j t}^{d}\left(n_{j t}^{s}, p_{i(t-)}^{d}\right)=\alpha_{j}^{d} n_{j t}^{s}+h_{j}^{d} p_{i(t-1)}^{d} \\
p_{j t}^{s}\left(n_{j t}^{d}, p_{i(t-)}^{s}\right)=\alpha_{j}^{s} n_{j t}^{d}+h_{j}^{s} p_{i(t-1)}^{s}
\end{array}\right.\right.
$$

Profit function of platforms can be transformed into

$$
\left\{\begin{array}{l}
\pi_{i}=\left(\alpha_{i}^{d} n_{i t}^{s}+h_{i}^{d} p_{j(t-1)}^{d}-c_{i}^{d}\right) n_{i t}^{d}+\left(\alpha_{i}^{s} n_{i t}^{d}+h_{i}^{s} p_{j(t-1)}^{s}-c_{i}^{s}\right) n_{i t}^{s}-C_{i} \\
\pi_{j}=\left(\alpha_{j}^{d} n_{j t}^{s}+h_{j}^{d} p_{i(t-1)}^{d}-c_{j}^{d}\right) n_{j t}^{d}+\left(\alpha_{j}^{s} n_{j t}^{d}+h_{j}^{s} p_{i(t-1)}^{s}-c_{j}^{s}\right) n_{j t}^{s}-C_{j}
\end{array}\right.
$$

Proposition 2: In the game about expected price, the price of one side set by platforms for users is determined by cross network externalities and expected adjustment of competitors' price. Final equilibrium results are 


$$
\left\{\begin{array} { l } 
{ n _ { i t } ^ { d } = \frac { c _ { i } ^ { s } - h _ { i } ^ { s } p _ { j ( t - 1 ) } ^ { s } } { a _ { i } ^ { d } + a _ { i } ^ { s } } } \\
{ n _ { i t } ^ { s } = \frac { c _ { i } ^ { d } - h _ { i } ^ { d } p _ { j ( t - 1 ) } ^ { d } } { a _ { i } ^ { d } + a _ { i } ^ { s } } } \\
{ n _ { j t } ^ { d } = \frac { c _ { j } ^ { s } - h _ { j } ^ { s } p _ { i ( t - 1 ) } ^ { s } } { a _ { j } ^ { d } + a _ { j } ^ { s } } } \\
{ n _ { j t } ^ { s } = \frac { c _ { j } ^ { d } - h _ { j } ^ { d } p _ { i ( t - 1 ) } ^ { d } } { a _ { j } ^ { d } + a _ { j } ^ { s } } }
\end{array} \left\{\begin{array}{l}
p_{i t}^{d}=\frac{a_{i}^{d} c_{i}^{d}+a_{i}^{s} h_{i}^{d} p_{j(t-1)}^{d}}{a_{i}^{d}+a_{i}^{s}} \\
p_{i t}^{s}=\frac{a_{i}^{s} c_{i}^{s}+a_{i}^{d} h_{i}^{s} p_{j(t-1)}^{s}}{a_{i}^{d}+a_{i}^{s}} \\
p_{j t}^{d}=\frac{a_{j}^{d} c_{j}^{d}+a_{j}^{s} h_{j}^{d} p_{j(t-1)}^{d}}{a_{j}^{d}+a_{j}^{s}} \\
p_{j t}^{s}=\frac{a_{j}^{s} c_{j}^{s}+a_{j}^{d} h_{j}^{s} p_{j(t-1)}^{s}}{a_{j}^{d}+a_{j}^{s}}
\end{array}\right.\right.
$$

From these results, the users' scale of one side on the platform is decided by the marginal cost of providing these service, prior price set by competitors and cross network externalities. The price of one side on the platform is depended on cross network externalities, the expected price of competitors and marginal cost.

(1) Cross network externalities coefficient. Assuming that the expected price of competitors and marginal cost are constant, the price function is

$$
p_{i}^{d}=\frac{a_{i}^{d} \overline{c_{i}^{d}}+a_{i}^{s} \overline{h_{i} p_{j(t-1)}^{d}}}{a_{i}^{d}+a_{i}^{s}},\left(\text { where } h_{i}>0\right) .
$$

When $\left(a_{i}^{d} \overline{c_{i}^{d}}+a_{i}^{s} \overline{h_{i} p_{j(t-1)}^{d}}\right)>\left(a_{i}^{d}+a_{i}^{s}\right)$, the larger the cross network externalities coefficient, the lower the price set by platforms. When $\left(a_{i}^{d} \overline{c_{i}^{d}}+a_{i}^{s} \overline{h_{i} p_{j(t-1)}^{d}}\right)<\left(a_{i}^{d}+a_{i}^{s}\right)$, the larger the cross network externalities coefficient, the higher the price set by platforms.

(2) Marginal cost. Assuming that cross network externalities coefficient and the expected price of competitors are constant, the price function is

$$
p_{i}^{d}=\frac{\overline{a_{i}^{d}} c_{i}^{d}+\overline{a_{i}^{s}} \overline{h_{i} p_{j(t-1)}^{d}}}{\overline{a_{i}^{d}}+\overline{a_{i}^{s}}}
$$

It means that the higher the cost of these services provided for each customer interaction, the higher the price charged by platforms.

(3) Expected price of competitors. Assuming that cross network externalities coefficient and marginal cost are constant, the price function is

$$
p_{i}^{d}=\frac{\overline{a_{i}^{d}} \overline{c_{i}^{d}}+\overline{a_{i}^{s}} h_{i} p_{j(t-1)}^{d}}{\overline{a_{i}^{d}}+\overline{a_{i}^{s}}},\left(\text { where } h_{i}>0\right) .
$$

It means that the price set by platforms is a positive correlation with the expected price on the other side of competing platforms.

Also it is known that platforms can set a price on their service based on the size of externalities, and then enter the competitive differentiation stage, after critical capacity. 
Corollary 2: In repeated games, the equilibrium price of internet platforms is

$$
p_{i t}^{d}=\frac{\left(a_{i}^{d} c_{i}^{d}+a_{i}^{s} h_{i}^{d}\right) a_{j}^{d} c_{j}^{d}+\left(a_{j}^{d}+a_{j}^{s}\right) a_{i}^{d} c_{i}^{d}}{\left(a_{i}^{d}+a_{i}^{s}\right)\left(a_{j}^{d}+a_{j}^{s}\right)-\left(a_{i}^{d} c_{i}^{d}+a_{i}^{s} h_{i}^{d}\right) a_{j}^{s} h_{j}^{d}} .
$$

This article analyzes the competitive strategies between the two platforms. To simplify the analysis, the interaction coefficient of the same platform does not distinguish, namely $a_{i}^{d}=a_{i}^{s}, a_{j}^{d}=a_{j}^{s}$. Thus, the formula can be simplified to:

$$
p_{i}=\frac{2\left(c_{i}+h_{i}\right) a_{i} a_{j} c_{j}+2 a_{j} a_{i} c_{i}}{4 a_{i} a_{j}-2\left(c_{i}+h_{i}\right) a_{i} a_{j} h_{j}}=\frac{\left(c_{i}+h_{i}\right) c_{j}+c_{i}}{2-\left(c_{i}+h_{i}\right) h_{j}} .
$$

The price of the platforms is affected by the marginal cost and expected adjustment factor.

According to adaptive expectations, the expected price is mainly affected by the previous one, that is $h_{i}=h_{j}=1$, the price function can be translated into

$$
p_{i}=\frac{\left(c_{i}+1\right) c_{j}+c_{i}}{2-\left(c_{i}+1\right)^{*} 1}=\frac{c_{i} c_{j}+c_{j}+c_{i}}{1-c_{i}}
$$

Partial derivative:

$$
\left\{\begin{array}{l}
\frac{\partial p_{i}}{\partial c_{i}}=\frac{1}{\left(1-c_{i}\right)^{2}} \\
\frac{\partial p_{i}}{\partial c_{j}}=\frac{1+c_{i}}{1-c_{i}}
\end{array} .\right.
$$

As

$$
\frac{\partial p_{i}}{\partial c_{i}}>0
$$

the price of a platform is a positive correlation with its marginal cost, namely the higher the marginal cost of the platforms, the higher the tariffs will be charged. As for the effect of others' cost, when $c_{i}>1$ the price of a platform is a negative correlation with marginal cost of competitors; when $c_{i}<1$ the price of a platform is a positive correlation with marginal cost of competitors. This means platforms have the character of critical capacity.

\section{CONCLUSION}

According to our analysis, internet platforms are mainly based on the size of the network externalities to conduct game competition [14]. There is a critical mass in the development and competition of platforms [15]. Before the critical mass, the role of external effects of the platform is very small, so it pursues rapid expansion of the size of the users, which can survive and avoid the establishment of failure. After the critical mass, the role of external effects of the platform is larger, so it adopts difference competition based on the size and the expected external behavior of competitors, which is in order to maximize corporate profits.

Therefore, internet platforms need to compete based on the stage. 
First, before the critical mass, platforms can adopt a single product or services strategy in order to control costs, as much as possible to attract more customers. It uses cost minimization rather than differentiation strategy to pursuit rapid growth in user scale.

Second, after reaching critical mass, platforms can adopt a differentiation strategy based on network externalities size. It can better meet customers' different demands by providing different products or services for different customer groups, according to interactive coefficient.

\section{ACKNOWLEDGEMENTS}

The author is highly grateful to any anonymous referee for their careful reading and insightful comments, and the views and opinions expressed are those of the authors. The work is supported by the National Social Science Foundation of China (No. 14CGL012) and National Science and Technology Support Program (No. 2012BAH69F03).

\section{REFERENCES}

[1] Rochet, J.C. \& Tirole, J., Cooperation among competitors: some economics of payment card associations. RAND Journal of Economics, 33(4), pp. 549-570, 2002. http://dx.doi.org/ $10.2307 / 3087474$

[2] Jullien, B.C.A.B., Chicken \& egg: competition among intermediation service providers. RAND Journal of Economics, 34(2), pp. 309-328, 2003. http://dx.doi.org/10.2307/1593720

[3] Hagiu, A., Pricing and commitment by two-sided platforms. RAND Journal of Economics, 37(3), pp. 720-737, 2006.

[4] Shapiro, C. \& Varian, H.R., Information rules: a strategic guide to the network economy. Harvard Business Review, pp. 152-198, 1999, ISBN: 7-300-03493-4/F.1048

[5] Katz, M.L. \& Shapiro, C., Network externalities, competition, and compatibility. American Economic Review, 3(75), pp. 424-440, 1985.

[6] Rochet, J. \& Tirole, J., Platform competition in two-sided markets. Journal of the European Economic Association, 1(4), pp. 990-1029, 2003. http://dx.doi.org/10.1162/154247603322493212

[7] Armstrong, M., Competition in two-sided markets. RAND Journal of Economics, 37(3), pp. 668-691, 2006.

[8] Economides, N., The economics of networks. International Journal of Industrial Organization, 14(2), pp. 1-33, 1996.

[9] Rochet, J. \& Tirole, J., Two-sided markets: a progress report. RAND Journal of Economics, 37(3), pp. 645-667, 2006. http://dx.doi.org/10.1111/j.1756-2171.2006.tb00036.x

[10] Weyl, E.G., A price theory of multi-sided platforms. American Economic Review, 100, pp. 1642-1742, 2010. http://dx.doi.org/10.1257/aer.100.4.1642

[11] Weyl, E.G. \& White, A., Insulated platform competition. Working Paper, 2013, available at SSRN: http://dx.doi.org/10.2139/ssrn.1694317.

[12] Wright, J., The determinants of optimal interchange fees in payment systems. Journal of Industrial Economics, 52, pp. 1-26, 2004. http://dx.doi.org/10.1111/j.0022-1821.2004.00214.x

[13] Fudenberg, D. \& Peysakhovich, A., Recency, records and recaps: learning and non-equilibrium behavior in a simple decision problem, Working Paper, 2014, available at SSRN: http://dx.doi .org/10.2139/ssrn.2339862

[14] Rysman, M., The economics of two-sided markets. Journal of Economic Perspectives, 23(3), pp. 125-143, 2009. http://dx.doi.org/10.1257/jep.23.3.125

[15] Evans, D.S., Platform economics: essays on multi-sided businesses. Competition Policy International, 2011. 